\title{
Hollow microsphere Fabry-Perot cavity for sensing applications
}

\author{
Catarina Monteiro, Susana Silva, Orlando Frazão
}

\begin{abstract}
Two different sensing structures based on a hollow microsphere Fabry-Perot cavity are proposed. The hollow spheroidal cavities are fabricated resorting only to fusion splicing. The first structure is based on a hollow microsphere located at the fiber end and works as a probe sensor. The structure was subjected to lateral load pressure and presents a sensitivity of $1.56 \pm 0.01 \mathrm{~nm} / \mathrm{N}$. The second proposed sensor relies on an in-line hollow microsphere. The sensing structure allows the detection of lateral loading, with a sensitivity of $2.62 \pm 0.02 \mathrm{~nm} / \mathrm{N}$, as well as strain detection, with a sensitivity of $4.66 \pm 0.03 \mathrm{pm} / \mu \varepsilon$. The two proposed sensors present similar response when subjected to temperature and have low thermal sensitivity.
\end{abstract}

Index Terms - Fabry-Perot Interferometry, Fiber Optics, Lateral Loading, Optical Sensors, Strain, Temperature.

\section{INTRODUCTION}

$\mathrm{O}$ PTICAL fiber sensors based on interferometric configurations have been extensively studied in the last decades and have found many applications in physical and chemical sensing. The most explored configurations are the ones based on Mach-Zehnder [1], Sagnac [2], Michelson [3] and finally, low finesse Fabry-Perot [4] interferometers. The low finesse Fabry-Perot is a very simple structure that results from the interference between two or more waves that go through different optical paths with different reflectivity in the sensor. The sensing applications for this configuration are vast, ranging from strain [5], pressure [6], magnetic field [7] and temperature [8]. Cavity fabrication can be achieved by using chemical etching [9], fiber Bragg Gratings (FBGs) serving as mirrors [10], micromachining [11] or by the use of fusion splicing [12]. The fusion splicing technique differentiates itself as an easy, fast and low cost fabrication method without chemical hazards.

Fabry-Perot cavities fabricated resorting only to fusion splicing have been achieved by using photonic crystal fibers (PCFs) [13] or simple standard optical fibers [14]-[16].

In this work, two different sensing structures based on a hollow microsphere cavity are proposed. The first sensor

Manuscript received ...

The authors are with the Instituto de Engenharia de Sistemas e Computadores, Tecnologia e Ciência, Porto 4169-007, Portugal, and also with the Department of Physics and Astronomy, Faculty of Sciences, University of Porto, Porto 4169-007, Portugal (e-mail:ofrazao@inescporto.pt).

Color versions of one or more of the figures in this letter are available online at http://ieeexplore.ieee.org. structure proposed is based on a hollow microsphere tip. This sensor shows great sensitivity to lateral load with very low thermal sensitivity. Subsequently, an in-line sensor structure with microbubble cavity is presented. This configuration yields a simpler reflected spectrum as the Fabry-Perot cavity can be treated as a two-wave interferometer. The sensor, besides being highly sensitive to lateral loading with low thermal sensitivity, presents the possibility to be subjected to strain.

\section{SENSOR FABRICATION}

The proposed sensor fabrication involves only fusion splicing and cleaving processes, using three different manual splicing programs, schematized in Table 1. In the first step, presented in Fig. 1(a), a cleaved single mode fiber (SMF) was fusion spliced to a hollow core fiber (or silica capillary tube) using the manual Program 1- Table I. The electric arc was centered on the SMF to prevent the collapse of the hollow core fiber due to the heat of the fusion splicing process, while being able to join the two fibers. The resulting configuration was, then, cleaved to the desired length, as schematized in Fig. 1(b). This length controls the cavity length as well as the shape of the hollow microsphere. Applying two consecutive electric arcs at the tip of the hollow core fiber the microsphere was formed, as

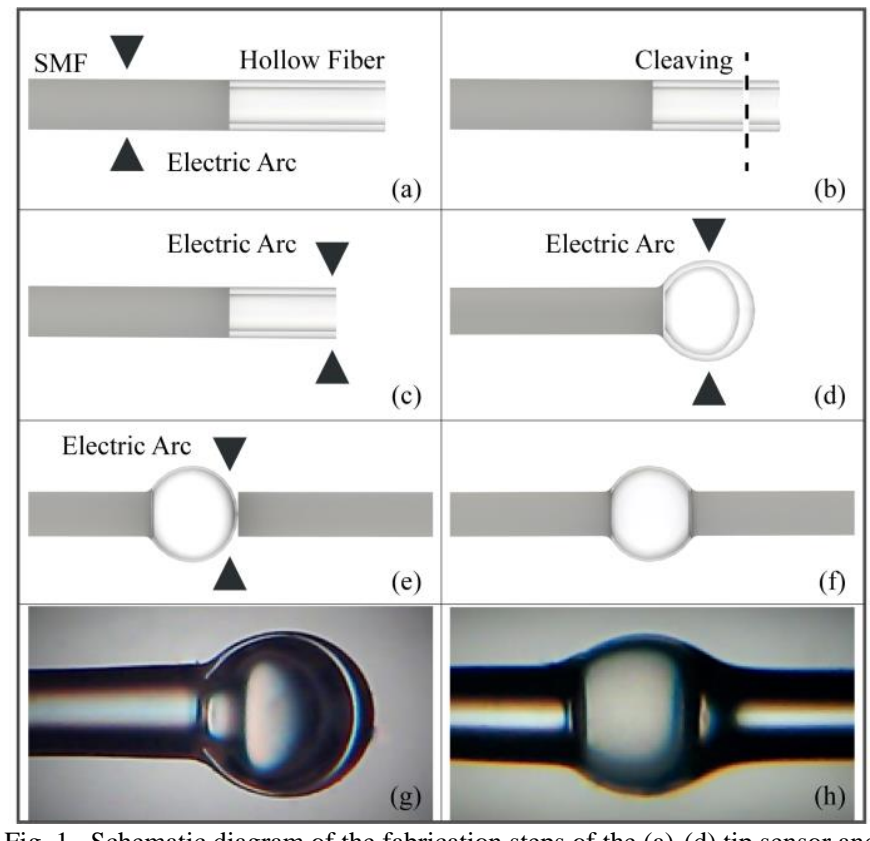

Fig. 1. Schematic diagram of the fabrication steps of the (a)-(d) tip sensor and (a)-(f) in-line sensor. Microscope photograph of (g) tip sensor and (h) in-line. sensor. 
TABLE I

MANUAL SPLICING PARAMETERS

\begin{tabular}{cccc}
\hline \hline Parameter & Program 1 & Program 2 & Program 3 \\
\hline Pre-fusion Time (s) & 0 & 0 & 0 \\
Fusion Time (ms) & 300 & 2000 & 600 \\
Arc Power* & S-075 & S+100 & S-050 \\
\hline \hline
\end{tabular}

* Absolute arc-power was not possible to attain; only relative values were attained ( $\mathrm{S}$ being the standard value).

shown in Fig. 1(c) and (d), using Program 2 - Table I. The first electric arc results in the formation of a hollow microsphere with thick silica walls, while the second electric arc narrows the silica walls. In Fig. 1(g) is depicted a microscope photograph of the resultant structure. The fabricated sensor presents a $\sim 175 \mu \mathrm{m}$ air cavity with a $\sim 30 \mu \mathrm{m}$ silica wall. The sensor tip presents two optical cavities: the first is an air cavity and the second is a silica cavity formed on the outer wall. The resultant reflected signal, shown in Fig. 2(a), is the outcome of the interference of the three reflected waves that arises from the three interfaces in the sensor head. The first reflection occurs in the interface between the SMF and the air cavity and the second reflection arises in the interface between air and the silica wall. The third reflection results from the refraction index changes in the interface between the silica wall and the surrounding media from outside of the sensing head. Three different interferences occur: between the first and the second wave, between the first and the third wave, and between the second and the third wave. Due to the small cavity length provided by the silica wall, the interference between the second and the third wave may be neglected. The remaining interferences are dominant and their interaction results in a beat, as presented in Fig. 2(a). The

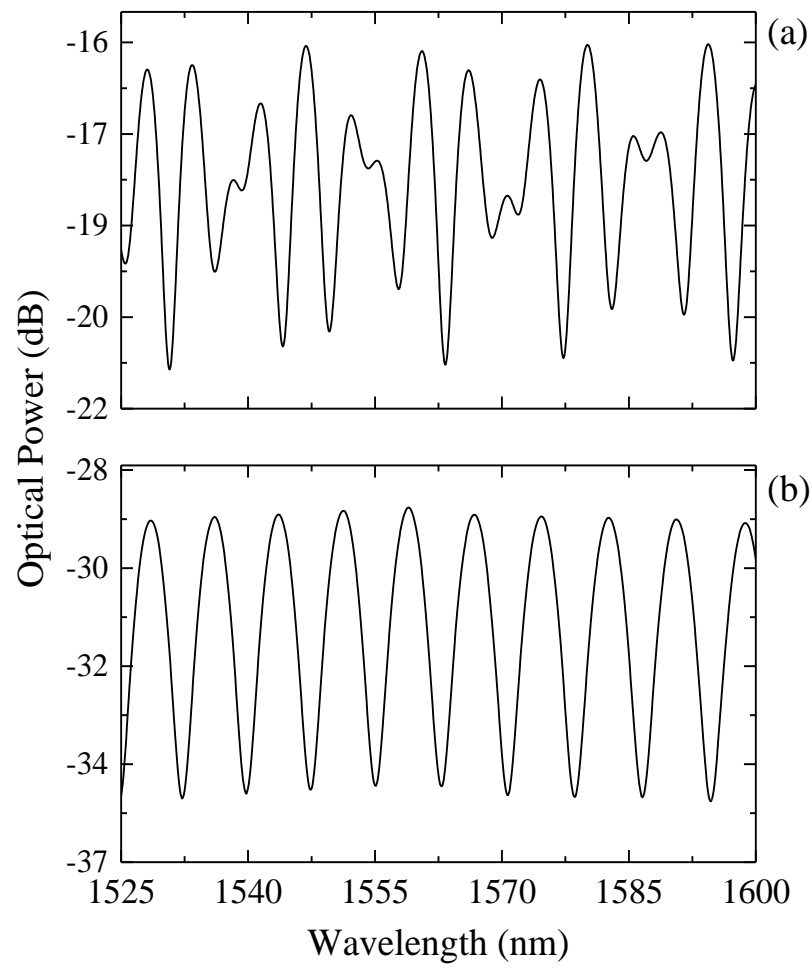

Fig. 2. Reflected spectrum of the (a) tip sensor, and (b) in-line sensor.

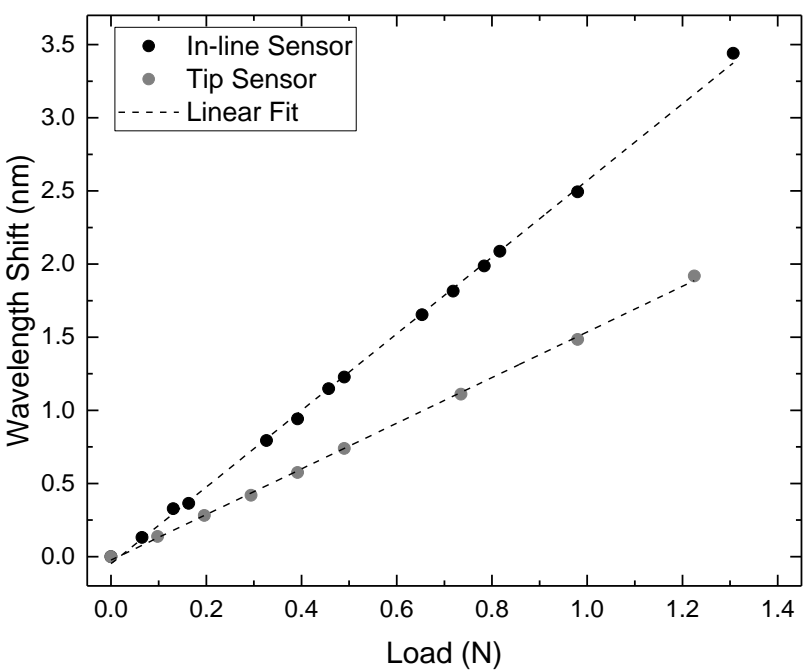

Fig. 3. Lateral loading response for the tip and the in-line sensor.

hollow microsphere tip sensor structure allows the detection of transverse loading [17], and refractive index [18].

In a second approach, an in-line configuration was fabricated by splicing a cleaved SMF to the tip of the hollow microsphere, using Program 3 - Table I. This configuration, presented in Fig. 1(b), allows strain sensing, while maintaining lateral loading sensitivity. The resultant reflected spectrum, presented in Fig. 2(b), is a two wave interferometer. In this case, the thickness of the microspheres is negligible when compared with the first structure, so the interference occurs between the reflected wave in the interface SMF/air and the reflected wave from the air/silica interface.

\section{EXPERIMENTAL RESULTS}

The fabricated sensors were tested using a reflection interrogation system composed by a broadband optical source, an optical circulator and an Optical Spectrum Analyzer (OSA). The optical source had a bandwidth of $100 \mathrm{~nm}$, with a central operation wavelength at $1550 \mathrm{~nm}$.

Lateral loading response was tested for the two sensor structures using a three-point application setup in order to

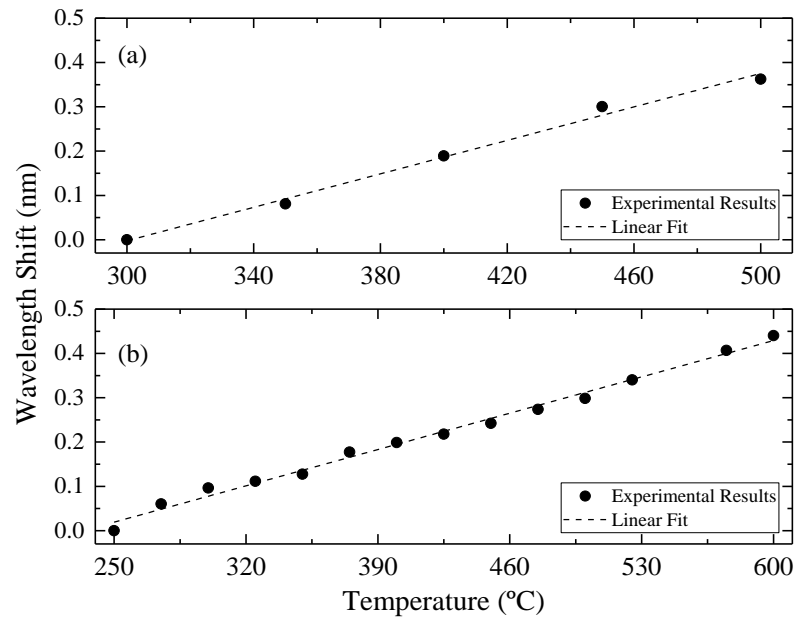

Fig. 4. Temperature response of the (a) tip sensor and (b) in-line sensor. 


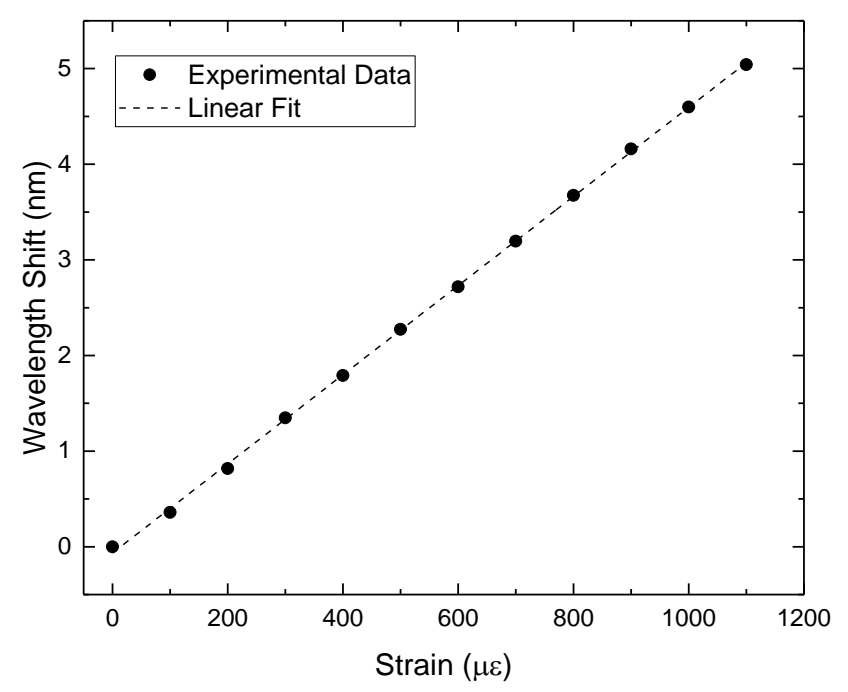

Fig. 5. Strain response of the in-line sensor.

equally divide the applied load. The load response for the two structures is presented in Fig. 3. The sensor tip structure yield a $1.56 \pm 0.01 \mathrm{~nm} / \mathrm{N}$ sensitivity. This result was at the same order of magnitude than the one previously reported in literature [17] for similar structures. Regarding the in-line structure, a sensitivity of $2.62 \pm 0.02 \mathrm{~nm} / \mathrm{N}$ was attained. This higher sensitivity may be due to the thinner walls presented in the inline hollow microsphere structure that will result in higher deformation to the applied load. Temperature response was also attained for the two structures, as presented in Fig. 4. The sensor tip was tested for temperatures in the range of 300 to $500{ }^{\circ} \mathrm{C}$, and a sensitivity of $1.90 \pm 0.06 \mathrm{pm} /{ }^{\circ} \mathrm{C}$ was attained. In the inline configuration, temperatures varied between 250 and $600{ }^{\circ} \mathrm{C}$ and presents a linear response with a sensitivity of $1.17 \mathrm{pm} /{ }^{\circ} \mathrm{C}$. Thermal expansion of the silica microsphere is the dominant behavior responsible for the shifting of the spectrum signal to higher wavelengths. The sensors presented a smaller temperature sensitivity when compared to similar structures, proposed by Ma et al. [17] and Ferreira et al. [19], and presented a sensitivity of the same order of magnitude to the achieved by Liu et al. [14]. For the first structure, the crosssensitivity between lateral loading and temperature is $1 \times 10^{-3}$ $\mathrm{N} /{ }^{\circ} \mathrm{C}$ and, for the second structure, a lower cross-sensitivity of $0.75 \times 10^{-3} \mathrm{~N} /{ }^{\circ} \mathrm{C}$ was achieved.

The in-line sensor was tested for strain, in a range of $1000 \mu \varepsilon$. As presented in Fig. 5, a linear response was attained with a sensitivity of $4.66 \pm 0.03 \mathrm{pm} / \mu \varepsilon$. The cross-sensitivity between strain and temperature was $0.25 \mu \varepsilon /{ }^{\circ} \mathrm{C}$. A structure with an array of silica microspheres was proposed by Ferreira et al. [19], where a maximum sensitivity of $-1.59 \mathrm{pm} / \mu \varepsilon$ was achieved, almost 3 times smaller than the sensor proposed in this work. Liu et al. [15] reported an all-fiber Fabry-Perot structure and a $2.9 \mathrm{pm} / \mu \varepsilon$ sensitivity was achieved for $79 \mu \mathrm{m}$ cavity length, which is much smaller than the one obtained in the present case.

\section{CONCLUSIONS}

Two sensing structures based on a hollow microsphere Fabry-Perot cavity were proposed. The first configuration was based on a hollow microsphere fiber tip, which was tested for lateral loading and temperature. This sensing structure yielded high lateral loading sensitivity, namely $1.56 \pm 0.01 \mathrm{~nm} / \mathrm{N}$, and a low thermal cross-sensitivity of $1 \times 10^{-3} \mathrm{~N} /{ }^{\circ} \mathrm{C}$.

The second device was an in-line sensor fabricated by fusion splicing a SMF to a hollow microsphere structure. The proposed sensor was tested for lateral loading and strain, presenting sensitivities of $2.62 \pm 0.02 \mathrm{~nm} / \mathrm{N}$ and $4.66 \pm 0.03 \mathrm{pm} / \mu \varepsilon$, respectively. The higher sensitivity to lateral loading may be due to the smaller outer silica walls when compared to the hollow microsphere-based sensor tip. This structure also presented a low thermal cross-sensitivity of $0.75 \times 10^{-3} \mathrm{~N} /{ }^{\circ} \mathrm{C}$, and a low cross-sensitivity between strain and temperature. The presented in-line structure may be suitable for lateral loading and strain sensing in harsh environments.

\section{ACKNOWLEDGMENT}

This work was financed by the FCT, Fundação para a Ciência e Tecnologia (Portuguese Foundation for Science and Technology) and by the ERDF (European Regional Development Fund) through COMPETE Programme (Operational Programme for Competitiveness) within project FCOMP-01-0124-FEDER-037281. S. O. Silva received a PosDoc fellowship (ref. SFRH/BPD/92418/2013) also funded by the EU and the Portuguese State.

\section{REFERENCES}

[1] A. Dandridge and A. B. Tveten, "Phase compensation in interferometric fiber-optic sensors.," Opt. Lett., vol. 7, no. 6, pp. 279-281, 1982.

[2] E. Udd, "Fiber-Optic Acoustic Sensor Based on the Sagnac Interferometer," Proc. Soc. Photo-Optical Instrum. Eng., vol. 425, pp. 90-95, 1983.

[3] M. Imai, T. Ohashi, and Y. Ohtsuka, "High-sensitive all-fiber Michelson interferometer by use of differential output configuration," Opt. Commun., vol. 39, no. 1, pp. 7-10, 1981.

[4] J. S. Sirkis, D. D. Brennan, M. A. Putman, T. A. Berkoff, A. D. Kersey, and E. J. Friebele, "In-Line Fiber Etalon for Strain-Measurement," Opt. Lett., vol. 18, no. 22, pp. 1973-1975, 1993.

[5] M. S. Ferreira, P. Roriz, J. Bierlich, J. Kobelke, K. Wondraczek, C. Aichele, K. Schuster, J. L. Santos, and O. Frazão, "Fabry-Perot cavity based on silica tube for strain sensing at high temperatures," Opt. Express, vol. 23, no. 12, p. 16063, 2015.

[6] J. Ma, J. Ju, L. Jin, and W. Jin, "A Compact Fiber-Tip Micro-Cavity Sensor for High-Pressure Measurement," IEEE Photonics Technol. Lett., vol. 23, no. 21, pp. 1561-1563, 2011.

[7] R. Q. Lv, Y. Zhao, D. Wang, and Q. Wang, "Magnetic fluid-filled optical fiber Fabry-P??rot sensor for magnetic field measurement," IEEE Photonics Technol. Lett., vol. 26, no. 3, pp. 217-219, 2014.

[8] M. S. Ferreira, P. Roriz, S. O. Silva, J. L. Santos, and O. Frazão, "Next generation of Fabry-Perot sensors for high-temperature," Opt. Fiber Technol., vol. 19, no. 6 PART B, pp. 833-837, 2013.

[9] V. R. Machavaram, R. A. Badcock, and G. F. Fernando, "Fabrication of intrinsic fibre Fabry-Perot sensors in silica fibres using hydrofluoric acid etching," Sensors Actuators, A Phys., vol. 138, no. 1, pp. 248-260, 2007.

[10] Y. O. Barmenkov, D. Zalvidea, S. Torres-Peiró, J. L. Cruz, and M. V. Andrés, "Effective length of short Fabry-Perot cavity formed by uniform fiber Bragg gratings," Opt. Express, vol. 14, no. 14, p. 6394, 2006.

[11] R. M. André, S. C. Warren-Smith, M. Becker, J. Dellith, M. Rothhardt, M. I. Zibaii, H. Latifi, M. B. Marques, H. Bartelt, and O. Frazão, "Simultaneous measurement of temperature and refractive index using focused ion beam milled Fabry-Perot cavities in optical fiber micro-tips," Opt. Express, vol. 24, no. 13, p. 14053, 2016.

[12] D. Jáuregui-Vázquez, J. M. Estudillo-Ayala, R. Rojas-Laguna, E. 
Vargas-Rodríguez, J. M. Sierra-Hernández, J. C. Hernández-García, and R. I. Mata-Chávez, "An all fiber intrinsic Fabry-Perot interferometer based on an air-microcavity," Sensors (Switzerland), vol. 13, no. 5, pp. 6355-6364, 2013.

[13] J. Villatoro, V. Finazzi, G. Coviello, and V. Pruneri, "Photonic-crystalfiber-enabled micro-Fabry-Perot interferometer.," Opt. Lett., vol. 34, no. 16, pp. 2441-2443, 2009.

[14] S. Liu, Y. Wang, C. Liao, G. Wang, Z. Li, Q. Wang, J. Zhou, K. Yang, $\mathrm{X}$. Zhong, J. Zhao, and J. Tang, "High-sensitivity strain sensor based on in-fiber improved Fabry-Perot interferometer," Opt. Lett., vol. 39, no. 7, pp. 2121-2124, 2014.

[15] C. Liao, S. Liu, L. Xu, C. Wang, Y. Wang, Z. Li, Q. Wang, and D. N. Wang, "Sub-micron silica diaphragm-based fiber-tip Fabry-Perot interferometer for pressure measurement," Opt. Lett., vol. 39, no. 10, p. 2827, 2014.

[16] S. Liu, K. Yang, Y. Wang, J. Qu, C. Liao, J. He, Z. Li, G. Yin, B. Sun,
J. Zhou, G. Wang, J. Tang, and J. Zhao, "High-sensitivity strain sensor based on in-fiber rectangular air bubble," Sci. Rep., vol. 5, no. Iii, p. 7624, 2015.

[17] J. Ma, J. Ju, L. Jin, W. Jin, and D. Wang, "Fiber-tip micro-cavity for temperature and transverse load sensing.," Opt. Express, vol. 19, no. 13, pp. 12418-12426, 2011.

[18] D. J. J. Hu, Y. Wang, J. L. Lim, T. Zhang, K. B. Mileńko, Z. Chen, M. Jiang, G. Wang, F. Luan, P. P. Shum, Q. Sun, H. Wei, W. Tong, and T. R. Woliński, "Novel miniaturized fabry-perot refractometer based on a simplified hollow-core fiber with a hollow silica sphere tip," IEEE Sens. $J .$, vol. 12, no. 5, pp. 1239-1245, 2012.

[19] M. S. Ferreira, J. L. Santos, and O. Frazão, "Silica microspheres array strain sensor," Opt. Lett., vol. 39, no. 20, pp. 5937-40, 2014. 